\title{
Roughness of sandpile surfaces
}

\author{
J. G. Oliveira, ${ }^{1}$ J. F. F. Mendes, ${ }^{1}$ and G. Tripathy ${ }^{2}$ \\ ${ }^{1}$ Departamento de Física, Universidade de Aveiro, Campus Universitário de Santiago, 3810-193 Aveiro, Portugal \\ ${ }^{2}$ Institute of Physics, Sachivalay Marg, Bhubaneswar 751005, India
}

(Received 19 November 2003; published 23 March 2004)

\begin{abstract}
We study the surface roughness of prototype models displaying self-organized criticality (SOC) and their noncritical variants in one dimension. For SOC systems, we find that two seemingly equivalent definitions of surface roughness yield different asymptotic scaling exponents. Using approximate analytical arguments and extensive numerical studies we conclude that this ambiguity is due to the special scaling properties of the nonlinear steady state surface. We also find that there is no such ambiguity for non-SOC models, although there may be intermediate crossovers to different roughness values. Such crossovers need to be distinguished from the true asymptotic behavior, as in the case of a noncritical disordered sandpile model studied by Barker and Mehta [Phys. Rev. E 61, 6765 (2000)].
\end{abstract}

DOI: 10.1103/PhysRevE.69.031105

PACS number(s): 05.40.-a, 05.70.Ln, 81.10.Aj

Since the original proposal by Bak, Tang, and Wisenfeld [1] there has been a large body of work directed towards understanding the ubiquity of scale invariance in externally driven open dissipative systems using the concept of selforganized criticality (SOC). The sandpile is a prototype model system which has been extensively used as a paradigm of SOC $[2,3]$. The principal aim has been to elucidate how slowly driven dissipative systems with fast relaxation mechanisms display long-tailed distributions of activity sizes. Recently, there has been a resurgance of interest in sandpile models in order to understand SOC in connection with better understood scale invariance in other nonequilibrium phenomena such as absorbing state phase transitions [4] and driven interfaces [5].

In Ref. [6], Krug, Socolar, and Grinstein (KSG) studied the surface fluctuations in a prototype model of SOC, the limited local sandpile (LLS) [3] and its variations in one spatial dimension and concluded that the interfacial fluctuations, although nontrivial, are evidently unconnected to the criticality of the system. In fact, they argued that the evolution of height fluctuations $\widetilde{h}(x, t)$ can be described by an extension of the Kardar-Parisi-Zhang (KPZ) $[7,8]$ equation for an anchored interface,

$$
\partial_{t} \widetilde{h}=D \partial_{x}^{2} \widetilde{h}+c \partial_{x} \widetilde{h}+\lambda\left(\partial_{x} \widetilde{h}\right)^{2}+\eta(x, t),
$$

where $\eta(x, t)$ is a Gaussian white noise. The linear term $c \partial_{x} \widetilde{h}$ is more relevant than the diffusion term $D \partial_{x}^{2} \widetilde{h}$ and the KPZ nonlinearity $\lambda\left(\partial_{x} \widetilde{h}\right)^{2}$ (in a renormalization group sense) and, since the interface is anchored, cannot be eliminated by a Galilean shift. This term is responsible for transporting fluctuations up the pile thus relating the spatial roughness of the anchored interface to the dynamical roughening of a moving KPZ interface in $d=1, \alpha_{L L S}=\beta_{K P Z(1 \mathrm{D})}=1 / 3$. This argument can be easily extended to higher dimensions.

In this paper we study interface roughness in LLS and related models and find that effect of the criticality of the system is somewhat subtle and is due to the nontrivial nature of the steady state surface. The value of surface roughness depends on whether it is measured about this nontrivial steady state profile or about the mean instantaneous surface, the latter being the correct choice for moving interfaces. The assertion made by KSG is valid only if $\tilde{h}$ in Eq. (1) is defined as the fluctuation around the nonlinear steady state surface. We also study modifications of the LLS which have nonlinear steady state profiles but which do not display SOC and find that there is no such ambiguity in the asymptotic roughness exponent. Thus, we conjecture that the roughness exponent is uniquely defined for systems not displaying SOC even though they may possess nontrivial steady state surfaces [9]. For SOC systems the ambiguity exists and one needs to define the roughness appropriately. Our results have important bearings on studies such as the recent one by Barker and Mehta [10] who observed an anomalously large roughness exponent in a sandpile model with structural disorder.

The limited local sandpile model in $d=1$ is defined as follows [see Fig. 1(a)]: on a one dimensional lattice of sites $i=0,1, \ldots, L$ we define an integer height variable $h_{i}$. One grain of sand added at a randomly chosen site increases the height at that site by $1: h_{i} \rightarrow h_{i}+1$. The configuration is stable if the local slopes satisfy $z_{i}=h_{i+1}-h_{i} \leqslant z_{c}$ for all $i$, where we chose $z_{c}=2$ (our results are essentially unchanged for $z_{c} \geqslant 2$ ). An instability occurs when by addition of grains at site $i+1$, the local slope exceeds the threshold $z_{i}>z_{c}$; in this case $z_{c}$ grains are transferred from column $i+1$ to column $i$. Subsequently, columns $i$ and $i+2$ may become unstable, setting off further topplings, leading to an avalanche. The grains involved in an avalanche leave the system if they reach site $i=0\left[h_{0}(t)=0, \forall t\right]$. A new grain is added after the system has attained a stable configuration. The final configuration reached is independent of the order in which the sites are updated in case more than one site become unstable. We count one unit of time (Monte Carlo step) for every $L$ grains added.

The standard way of quantifying avalanches in these systems is to count the number $m$ of grains that leave the pile after each deposition [3]. The criticality of the model is reflected in a broad probability distribution of avalanche sizes, $P(m)$ [Fig. 1(b)], which has no other length scale except the system size $L$. 

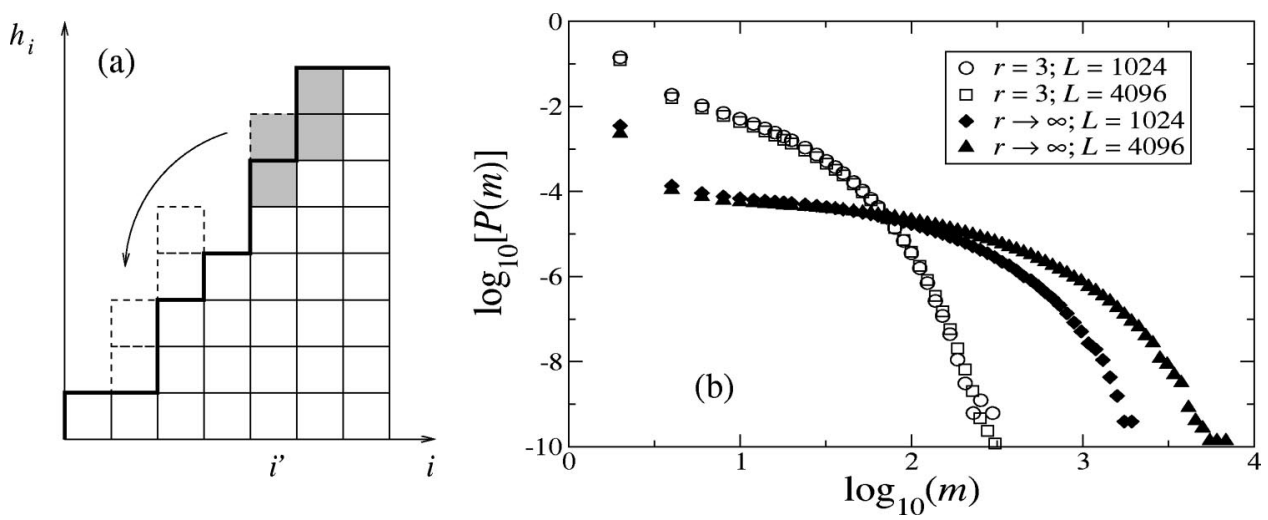

FIG. 1. (a) Dynamics of the sandpile: An initial surface (thick line), on becoming unstable, relaxes by transferring the unstable cluster (shaded blocks) successively to the left till a stable configuration is reached. (b) Log-log plot of the avalanche size distribution $P(m)$ for the LLS (solid symbols) and the ILLS (open symbols) for two different system sizes. $P(m)$ for the ILLS falls off with a characteristic length scale independent of $L$.

In Ref. [6], KSG also introduced and studied the inertial limited local sandpile (ILLS) which mimics the effect of inertia of the falling grains in a real sand pile. The instability condition setting off an avalanche is same as that in the LLS but the condition of stopping is changed. A cluster of grains when first destabilized is assigned a momentum $p=0$ and each time the front of the cluster reaches an unstable site $\left(z_{i}>z_{c}\right)$ it gathers momentum, $p \rightarrow p+1$. If it comes across a stable site, $p$ decreases by an amount $r: p \rightarrow p-r$. The cluster continues moving down as long as $p>0$ and leaves the pile if it reaches the site $i=0$. Clearly, $r=\infty$ corresponds to the LLS. It was noted by KSG that ILLS is not critical for any finite value of $r$ which is reflected in the corresponding avalanche size distribution [Fig. 1(b)].

To study the interfacial fluctuations in these models, we start with an initially flat pile $\left[h_{i}(0)=0, \forall i\right]$ and add grains till the pile reaches a steady state with the mean surface making a critical angle $\psi\left(\tan \psi_{L \rightarrow \infty}=3 / 2\right)$ with the horizontal. The closed boundary condition at $i=L$ ensures that the pile has only one surface with the critical slope [9]. The width of the interface, in the steady state, can be measured in two ways:

$$
\begin{aligned}
W_{1}^{2}(L) & =\frac{1}{L} \sum_{i=1}^{L}\left\langle\left[h_{i}(t)-\left\langle h_{i}\right\rangle\right]^{2}\right\rangle, \\
W_{2}^{2}(L) & =\frac{1}{L} \sum_{i=1}^{L}\left\langle\left[h_{i}(t)-s(t) i\right]^{2}\right\rangle,
\end{aligned}
$$

where $s(t)=2\left[\sum_{i=1}^{L} h_{i}(t)\right] / L(L+1)$ is the mean slope of the interface about which sum of instantaneous height fluctuations vanish $\sum_{i=1}^{L}\left[h_{i}(t)-s(t) i\right]=0$. In both definitions (2) above the ensemble average $\langle\cdots\rangle$ is identical to the time average in the steady state.

For large enough system sizes the scaling hypothesis is expected to be valid and the roughness exponent $\alpha$ is defined through

$$
W_{1,2}(L) \sim L^{\alpha}, \quad L \rightarrow \infty .
$$

In Fig. 2, we plot the two widths $W_{1}$ and $W_{2}$ for a range of system sizes $L=2^{n}$ with $6 \leqslant n \leqslant 14$ (that is $64 \leqslant L \leqslant 16384$ ). It is seen that the two widths have different asymptotic behaviors and give two different values of $\alpha$. The roughness $\alpha \simeq 0.33$, computed from $W_{1}$ (and $W_{2}$ up to $L \leqq L_{c}$ ), is in accordance with the predictions of Eq. (1). The asymptotic roughness computed from $W_{2}$, beyond the crossover system size $L_{c} \sim 1024, \alpha=0.65 \pm 0.02$, is larger than that for an interface generated by a simple random walk. This is surprising since, prima facie, the definitions in Eq. (2) are expected to be equivalent as far as asymptotic scaling is concerned [11]. In fact, for the ILLS, we have computed $W_{1}$ and $W_{2}$ for $r=20$ (Fig. 2, inset) and it is seen that they indeed have the same asymptotic behavior and hence, a unique value of $\alpha \simeq 0.33$.

We now show that the crossover in $W_{2}$ in the LLS can be traced to the fact that the time averaged steady state profile is not linear, i.e., $\left\langle h_{i}(t)\right\rangle \neq \bar{s} i$ and this makes the two defini-

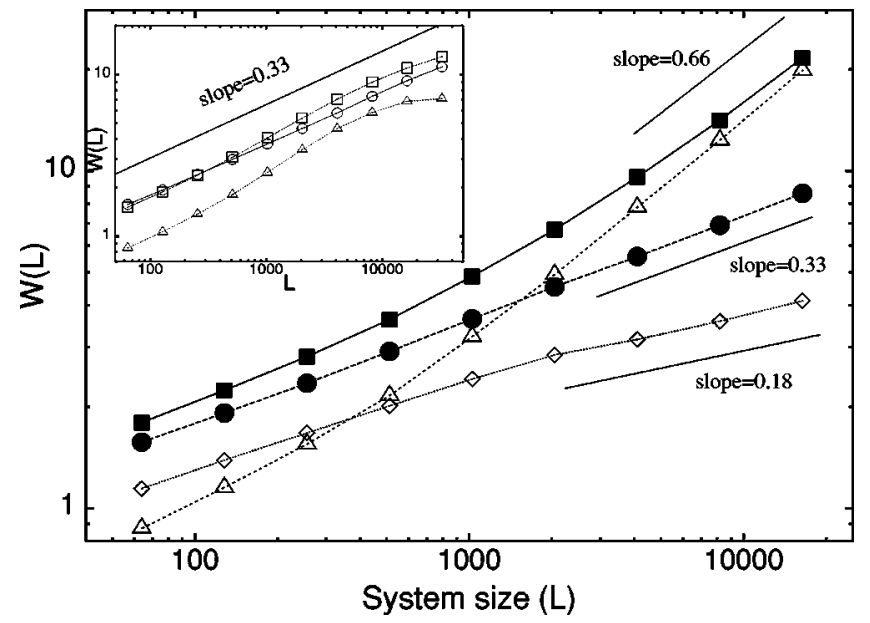

FIG. 2. Width vs system size for LLS: $W_{1}$ (circles) and $W_{2}$ (squares) are obtained using Eq. (2) while $W_{S}$ (triangles) is computed from Eq. (5) below. $W_{c}$ (diamonds) $\sim L^{0.18}$ asymptotically. Inset: $W_{1}$ (circles) and $W_{2}$ (squares) and $W_{S}$ (triangles) for the ILLS with $r=20$. 
tions nonequivalent. Although, this nonlinearity of $\left\langle h_{i}(t)\right\rangle$ is a consequence of the boundary effects and is present for the ILLS as well, we demonstrate that the presence of SOC in LLS results in special scaling properties which is responsible for the observed differences between asymptotic scalings of $W_{1}$ and $W_{2}$. It can be shown that $W_{1}$ and $W_{2}$ are related by

$$
W_{2}^{2}(L)+W_{c}^{2}(L)=W_{1}^{2}(L)+W_{S}^{2}(L),
$$

where $W_{S}(L)$ is the root-mean-square (rms) wandering of the steady state interface profile $\left\langle h_{i}\right\rangle$,

$$
W_{S}^{2}(L) \equiv \frac{1}{L} \sum_{i=0}^{L}\left[\left\langle h_{i}\right\rangle-\bar{s} i\right]^{2},
$$

with $\bar{s}=\langle s(t)\rangle$ being the mean slope of the steady state profile satisfying $\sum_{i=0}^{L}\left[\left\langle h_{i}\right\rangle-\bar{s} i\right]=0$. The second term on the left of $(4), W_{c}^{2}(L)=\left\langle[s(t)-\bar{s}]^{2}\right\rangle(L+1)(2 L+1) / 6$, is the contribution to the interface width due to instantaneous slope fluctuations and is the analog of the center of mass fluctuations in the case of moving interfaces [8]. In fact, in the case of moving interfaces $W_{c}$ dominates for large length scales, and hence $W_{2}$ is taken as the width. As it turns out, for the anchored interfaces we deal with here, $W_{c}$ is negligible compared to the other terms in Eq. (4) as $L \rightarrow \infty$. From Fig. 2, it is evident that the crossover in $W_{2}(L)$ for the LLS is due to the contribution from $W_{S}$. In the following we show that, for the LLS, the special form of the nonlinear steady state profile $\left\langle h_{i}(t)\right\rangle$, which results in $W_{S} \sim L^{0.66}$, is a consequence of the singular diffusion associated with the SOC state.

In Ref. [12], using a simple model, Carlson et al. demonstrated that the SOC state of the system is associated with the vanishing of the density of troughs concommittant with the divergence of the corresponding diffusion coefficient. The troughs are defined as the sites for which $z_{i} \leqslant 0$ so that, in the LLS, an avalanche necessarily stops at a trough (or leave the system at $i=0)$. On a coarse grained level we define a set of densities $\left\{\rho_{n}(x, t) ; n=-\infty, \ldots,-1,0,1,2\right\}$ where $\rho_{n}(x, t)$ denotes the local density of sites with $z_{i}=n$. It follows that the coarse grained local slope $z(x, t)$, which is locally conserved by the dynamics, may be expressed in terms of the $\rho_{n}$ 's as $z(x, t)=\sum_{n=-\infty}^{2} n \rho_{n}(x, t)$. The open boundary condition at $i=0$ implies $h(0, t)=0$, and the closed boundary condition at $i=L$ is modeled by setting $z(L, t)=0$. The trough density $\rho(x, t) \equiv \sum_{n=-\infty}^{0} \rho_{n}(x, t)$ is not strictly conserved. In the ' 01 ' model considered in Ref. [12] the slope has only two values $z_{i}=0$ (trough) and 1 , and, hence, both $z$ as well as $\rho$ are strictly conserved and are related simply as $z=1-\rho$. Although $\rho$ is not strictly conserved for LLS and ILLS we still approximate its dynamical evolution by the continuity equation, $\partial_{t} \rho(x, t)+\partial_{x} J[\rho(x, t)]=0$. Phenomenologically, the trough current $J$ consists of three parts: (i) current due to addition of grains $J_{0}$, (ii) avalanche current $J_{A}$, and (iii) a microscopic noise term $\eta(x, t)$. In analogy with driven diffusive systems [6] the phenomenological form of the avalanche current is written as $J_{A}(\rho)=a \rho+b \rho^{2}+\cdots$ $-D(\rho) \partial_{x} \rho$. As in the 01 model, the critical state of the system $(L \rightarrow \infty, r=\infty)$ is associated with $\rho, \partial_{x} \rho \rightarrow 0$ and

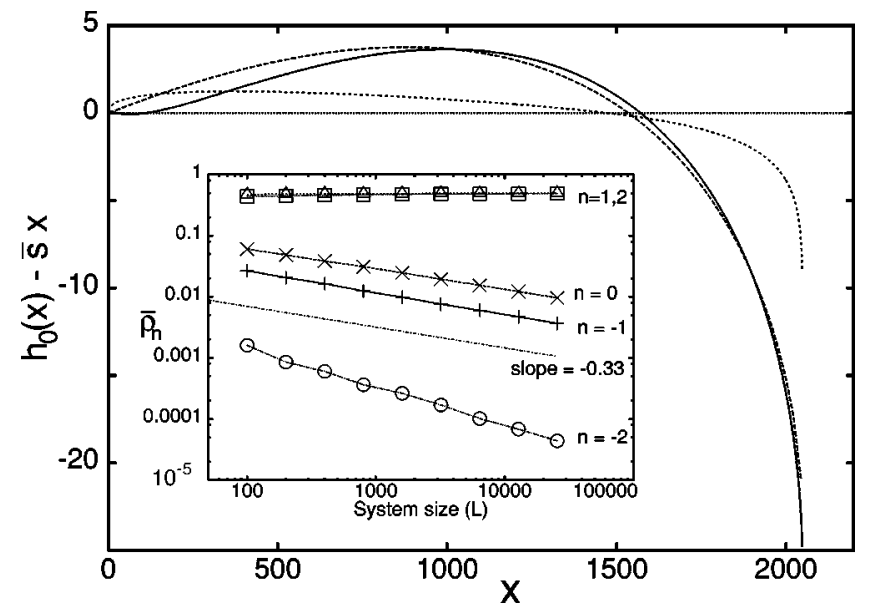

FIG. 3. Deviation of the steady state height profile from straight line. For the LLS, the simulation data (solid curve) are well approximated by the analytic form (8) (dashed curve) for $L=2048$ with optimally chosen values of parameters $\rho_{L}$ and $\gamma$. The dotted curve is for the ILLS with $r=20$. Inset: Average density $\bar{\rho}_{n}$ of sites with slope $z_{i}=n$ in the steady state as a function of system size $L$.

hence in order to balance the finite input flux $J_{0}$, the diffusion constant $D(\rho \rightarrow 0)$ has to diverge appropriately [12]. Without a loss of generality the leading divergence in $D$ is taken to be a simple pole and the evolution of $\rho(x, t)$ is thus [13]

$$
\frac{\partial \rho}{\partial t}=\frac{\partial}{\partial x}\left[D(\rho) \frac{\partial \rho}{\partial x}+\eta\right], \quad D(\rho)=\frac{A(\rho)}{\rho^{\phi}} .
$$

Here $A(\rho)$ is taken to be a smoothly varying function in the relevant interval $0 \leqslant \rho \leqslant 1$.

In order to compute the steady state density profile $\rho(x)$ $=\rho(x, t \rightarrow \infty)$ of troughs, we note that, as $L \rightarrow \infty$, the system is arbitrarily close to criticality $(\rho \rightarrow 0)$, and hence $A(\rho)$ may be set to a constant $A_{0}=A(0)$. One can then readily integrate the current equation $D(\rho) d \rho / d x=-J_{0}$, subjected to the boundary condition $\rho(L)=\rho_{L}$, to obtain

$$
\rho(x) \approx \rho_{L}[1+\gamma(L-x)]^{-\theta},
$$

where $\theta=1 /(\phi-1)$ and $\gamma=-J_{0} \rho_{L}^{1 / \theta} /\left(\theta A_{0}\right)$. The average density of the troughs $\bar{\rho} \equiv L^{-1} \int_{0}^{L} \rho(x) d x$ thus scales with system size asymptotically as $\bar{\rho} \simeq \rho_{L}(\gamma L)^{-\theta} /(1-\theta)$. From Fig. 3 (inset), we obtain $\theta \simeq 0.33$ and thus $\phi \simeq 4$ for the LLS, which are the same as the corresponding values obtained in Ref. [12] by direct measurement of $D(\rho)$ in a closed system.

In the following we find an approximate numerical relation between $\rho(x)$ to $z(x)$ which would enable us to find the steady state interface profile $h_{0}(x)=\int_{0}^{x} z(x) d x$, form (7). In Fig. 3 (inset), we plot the average densities $\bar{\rho}_{n}$ $\equiv L^{-1} \int_{0}^{L} d x\left\langle\rho_{n}(x, t)\right\rangle$ in the steady state as functions of the system size $L$. We note that, as $L$ increases, while $\bar{\rho}_{1} \simeq \bar{\rho}_{2}$ $\approx 1 / 2$ remain finite, densities of all the trough sites vanish algebraically with $L: \bar{\rho}_{0}, \bar{\rho}_{-1} \sim L^{-0.33}, \bar{\rho}_{-2} \sim L^{-0.6}$, and $\bar{\rho}_{n}$ 's with $n \leqslant-3$ are negligible. Hence, in the limit of large $L$, we 
can approximate the total trough density as $\bar{\rho} \simeq \bar{\rho}_{0}+\bar{\rho}_{-1}$. From the normalization condition $\sum_{n=-\infty}^{2} \rho_{n}(x, t)=1$ it follows that $\rho_{1}+\rho_{2}=1-\rho$ and we find numerically that $\bar{\rho}_{1}$ $-\bar{\rho}_{2} \simeq 0.46 \bar{\rho}$. Thus, for large systems, we may write $z(x)$ $\simeq 2 \rho_{2}+\rho_{1}-\rho_{-1} \simeq 3 / 2-\kappa \rho(x)$, where numerically $\kappa \simeq 2$ [14]. Thus, $h_{0}(x)$ is given approximately by

$$
h_{0}(x) \approx \frac{3}{2} x-\frac{\kappa \rho_{L}(1+\gamma L)^{1-\theta}}{\gamma(1-\theta)}\left(1-\left[\frac{1+\gamma(L-x)}{1+\gamma L}\right]^{1-\theta}\right)
$$

In Fig. 3, using the approximate expression for $\rho(x)$ from Eq. (7) with $\phi=4$, we compare $h_{0}(x)$ with that obtained numerically and notice that the agreement is rather good given the nature of the approximations involved [15].

The $L$ dependence of $W_{S}$ of the steady state profile can be computed from Eq. (8) and turns out to be

$$
W_{S}(L) \sim L^{1-\theta}
$$

For the LLS with $\theta \simeq 0.33, W_{S}(L) \sim L^{0.66}$ dominates $W_{1}$ $\sim L^{1 / 3}$ and $W_{c} \sim L^{0.18}$ in Eq. (4) and hence, for large $L$, $W_{2}(L) \sim L^{0.66}$-in very good agreement with our numerical results (Fig. 2). It is interesting to note that $W_{1}$ and $W_{2}$ would have different asymptotic behaviors if $\theta<2 / 3$, i.e., if $\phi>5 / 2$. For the 01 model studied in Ref. [12] it is shown that $\phi=3$ exactly and thus $W_{2}(L) \sim L^{1 / 2}$ for the corresponding interface [16]. Our conjecture that $W_{1}$ and $W_{2}$ would have different asymptotic behavior for SOC systems would be invalid if one can devise a model with $\phi<5 / 2$.

Next, we present a general argument as to why, for noncritical models such as the ILLS, we do not expect any ambiguity in the roughness exponent. The essential difference between critical and noncritical models is the presence of an additional length scale (apart from system size and the microscopic cutoff) which shows up in the avalanche size distribution (e.g., Fig. 1 for ILLS) as well as governs the decay of the boundary effects into the bulk. In the ILLS this length scale is related to the parameter $r$. To see this we first note that as a cluster moves down its momentum $p$ makes a random walk [6]. If the mean density of troughs $\rho<1 / r$ then most avalanches leave the system, resulting in net drainage; and if $\rho>1 / r$ then most avalanches stop on the pile, leading a to net growth of the pile. Thus, in the steady state one has a finite density of troughs $\rho=1 / r$ and thus the mean spacing between the troughs $1 / \rho=r$ appears as the additional length scale. As already discussed above, the current $J(\rho)$ now has a nonzero systematic part $a \rho+b \rho^{2} \ldots$, in addition to the finite diffusion term. Such a systematic current, e.g., a term such as $a \rho$, results in the effects of the boundaries decaying exponentially inside the bulk. This is in contrast to the long ranged power law decay in the LLS, which is reflected in the forms of $\rho(x)$ and $h_{0}(x)$. Although the steady state surface is nonlinear (Fig. 3), its rms wandering $W_{S}$ is bounded and does not alter the asymptotic scaling of $W_{2}$.

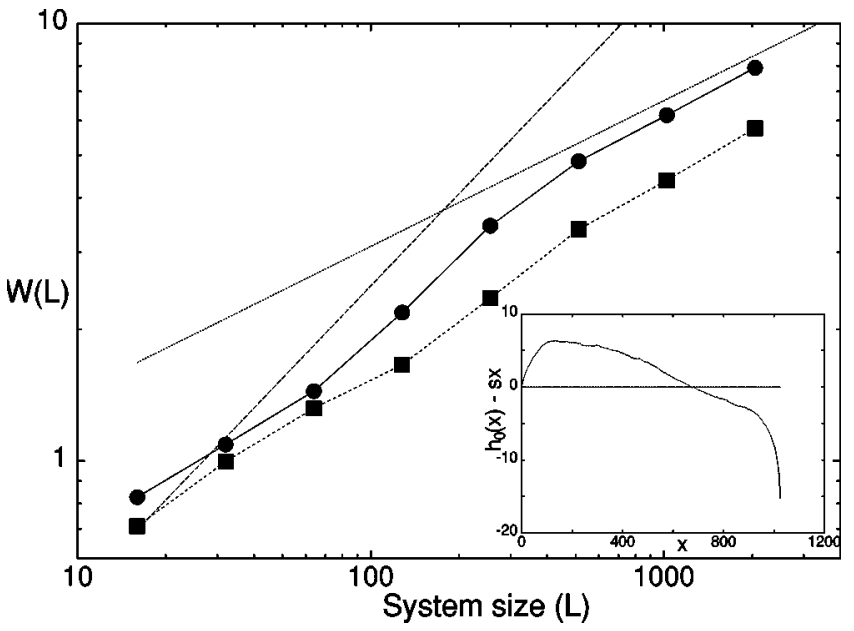

FIG. 4. Interface width of the disordered sandpile as a function of system size. The squares represent $W_{2}$ and circles represent $W$ $=\sqrt{W_{2}^{2}-W_{S}^{2}}$. The parameters chosen are the same as in Ref. [10]. The straight lines have slope 0.33 (dotted) and 0.72 (dashed). Inset: The steady state interface profile, after subtracting the mean linear profile.

Lastly, we briefly point out possible pitfalls of using $W_{2}$ naively without properly accounting for the underlying steady state profile, even in systems which do not show SOC. Recently, Barker and Mehta [10] studied a disordered version of the LLS where disorder was introduced by allowing the added grains to have an aspect ratio different from unity: grains are now rectangles and are deposited on the sandpile with fixed probabilities of landing on their larger or smaller edges. Thus, the height $h_{i}$ of column $i$, no longer an integer, is the sum of the vertical edges of all the grains in that column. In addition to the threshold dynamics of LLS, dynamical reorientation of cluster of grains were allowed. They found that the larger sandpiles cease to display SOC which is reflected in the emergence of a preferred size of the large avalanches in the associated drop number distributions. Their numerical studies of $W_{2}$ showed that while for very small systems $(L \lesssim 100)$ the roughness exponent $\alpha \simeq 0.34$, it seems to crossover to a much larger value $\alpha \simeq 0.72$ for larger system sizes, $100 \leqq L \lesssim 400$ (Fig. 4). Our preliminary numerical studies, using the same parameters as in Ref. [10], of yet larger systems $(500 \leqslant L \leqslant 2048)$ show that in fact the crossover seen in Ref. [10] is evidently transient and the asymptotic roughness exponent goes back to $\alpha \simeq 0.33$ (see Fig. 4).

In fact, the crossover reported in Ref. [10] is less noticeable if one looks at $W=\sqrt{W_{2}^{2}-W_{S}^{2}}$ instead of $W_{2}$ (Fig. 4) [17]. This is in accordance with our conjecture since the disordered system is not critical, although the steady state profile is nonlinear (Fig. 4, inset), it does not affect the asymptotic roughness measured by $W_{2}$. The transient crossover seen in $W_{2}$ here is similar to what is seen for the ILLS in Fig. 2, inset.

In summary, we have studied the surface roughness of a prototype model of SOC and its modifications in one dimension. We find that one needs to be careful in defining quantities such as the interface width, since the special form of 
the steady state shape of the surface in systems with SOC can result in different asymptotic behaviors of otherwise equivalent definitions. Although there is no such ambiguity for noncritical models, still there may be crossovers at intermediate length scales which should not be taken as the true asymptotic behavior.
J.G.O. and G.T. acknowledge financial support from Centro de Física do Porto, University of Porto, where part of this work was carried out. J.G.O. also acknowledges financial support from Universidade de Aveiro. J.F.F.M. was partially supported by Projects No. POCTI/1999/FIS/33141 and No. POCTI/2002/MAT/46176.
[1] P. Bak, C. Tang, and K. Wisenfeld, Phys. Rev. Lett. 59, 381 (1987); Phys. Rev. A 38, 364 (1988).

[2] Some reviews on the subject are H. J. Jensen, Self Organized Criticality (Cambridge University Press, Cambridge, 1998); D.L. Turcotte, Rep. Prog. Phys. 62, 1377 (1999); D. Dhar, Physica A 264, 1 (1999).

[3] L.P. Kadanoff, S.R. Nagel, L. Wu, and S.-M. Zhou, Phys. Rev. A 39, 6524 (1989).

[4] A. Vespignani, R. Dickman, M.A. Muñoz, and S. Zapperi, Phys. Rev. Lett. 81, 5676 (1998); Phys. Rev. E 62, 4564 (2000).

[5] M. Alava, J. Phys.: Condens. Matter 14, 2353 (2003); B. Tadic and D. Dhar, Phys. Rev. Lett. 79, 1519 (1997); C.-C. Chen and M. den Nijs, Phys. Rev. E 65, 031309 (2002); J.M. Luck and A. Mehta, Europhys. Lett. 54, 573 (2001).

[6] J. Krug, J.E.S. Socolar, and G. Grinstein, Phys. Rev. E 46, R4479 (1992).

[7] M. Kardar, G. Parisi, and Y.-C. Zhang, Phys. Rev. Lett. 56, 889 (1986).

[8] J. Krug, Adv. Phys. 46, 139 (1997).

[9] We exclude those situations in which the boundary conditions are such that the steady state profile has macroscopic regions of different slopes, e.g., as in a heap of sand with open boundary conditions at both ends in which the present discussions apply to each half of the pile separately. Also are excluded systems with quenched disorder, e.g., as in G. Tripathy and M. Barma, Phys. Rev. Lett. 78, 3039 (1997).

[10] G.C. Barker and Anita Mehta, Phys. Rev. E 61, 6765 (2000).

[11] However, as shown in Eq. (4), $W_{1}$ and $W_{2}$ are not numerically identical. E.g., for an anchored but flat surface which fluctuates as a whole $W_{2}=0$ but $W_{1} \neq 0$ whereas for a static nonplanar surface $W_{1}=0$ but $W_{2} \neq 0$.

[12] J.M. Carlson, J.T. Chayes, E.R. Grannan, and G.H. Swindle, Phys. Rev. Lett. 65, 2547 (1990); Phys. Rev. A 42, 2467 (1990).

[13] One could write the evolution equation for $z(x, t)$ directly as $\partial_{t} z(x, t)+\partial_{x} J(z, x, t)=0$, with the diffusion constant diverging as at $z=3 / 2$ for the LLS. However, for different models the critcal slope would be different from $3 / 2$, and hence it is more natural to write the equation for $\rho$ with the singularity always at $\rho=0$.

[14] We have numerically checked that, except for points close to the boundaries $x \simeq 0, L$, similar approximations hold good for the local densities $\rho_{n}(x, t)$ as for the spatially averaged ones.

[15] The deviations seem most pronounced near the left boundary $x=0$ which can be improved by including more terms in $A(\rho)=A_{0}+A_{1} \rho+\cdots$.

[16] J. G. Oliveira, J.F.F. Mendes, and G. Tripathy (unpublished).

[17] In the disordered case the slope fluctuations are large, and hence $W_{1}$ is dominated by $W_{c}$ and is numerically larger than $W_{2}$ in the range of system sizes we study. 